\title{
Improving the correct prescription and dosage of gentamicin
}

\author{
Dr. Imran Qureshi, Mr. Hani Habayeb, Dr. Clive Grundy,
}

\section{Abstract}

Gentamicin is an amino glycoside antibiotic, which is used predominantly in gram-negative infections but also has anti-staphylococcal activity. It is commonly used because of its lack of diffogenicity. There are recognised issues with its dosing of which time is a major factor.

\section{Problem}

Gentamicin dosing and prescription is often carried out incorrectly, despite very clear guidelines. Gentamicin is one of the few antibiotics which is weight-dependent in adults. The weight at which gentamicin needs to be prescribed is the patient's own body weight. This changes for obese individuals, where it must be given according to a corrected body weight, which involves a series of mathematical calculations. These calculations involve knowing the patient's height and weight and this can be a significant challenge, especially if the patient is bed-bound. An additional problem is that the gentamicin prescribing regime is relative to the patient's renal function. An accurate and acceptable measure of a patient's renal function is their creatinine clearance $(\mathrm{CrCl})$. $\mathrm{CrCl}$ is not a measure which is provided with the rest of the blood results and it must be manually calculated. Most prescribers use eGFR as a substitute although it should not be used for its relative inaccuracy. The height and weight of patients is nearly always guessed or approximated which means that patients can be severely underdosed or overdosed.

\section{Background}

Gentamicin is an antibiotic from the class of aminoglcosides. It is used predominantly to treat gram-negative infections, particularly urinary, biliary and abdominal but does have some anti-staphylococcal activity as well. Gentamicin is being used increasingly in clinical care because of its lack of 'diffogenicity' (causing clostridium difficile) but there are a number of problems associated with it. Gentamicin is a potentially nephrotoxic and ototoxic antibiotic if given incorrectly. A patient is given a dose according to their body weight. If they are obese, they are dosed according to a corrected body weight. The patient's renal function will also determine how many $\mathrm{mg} / \mathrm{kg}$ they will be dosed at. Once a patient is given gentamicin and is due to receive subsequent doses, they must have a level taken 6-14 hours after the initial dose which will demonstrate how well their kidneys are clearing the antibiotic as it is renally excreted. This manner of giving gentamicin is the once-daily extended interval regime which is replacing the traditional method of giving it more than once a day depending on peak and trough levels. It is presumed that this is a safer and more effective way of giving the antibiotic. Calculating the correct dose of gentamicin is dependent upon calculating the patient's ideal body weight, followed by the corrected body weight and finally their creatinine clearance. By not following this protocol significant variation can occur in dosing and subsequent prescription.

\section{Baseline}

For this project fifty patients from the surgical wards who had been prescribed gentamicin had their doses checked. Patients who had been given a prophylactic dose of $80-120 \mathrm{mg}$ were not included in the baseline measurement as there was no dependence on height, weight and renal function. For all of the patients, their height, weight, age, gender and serum creatinine at the time of dosing were recorded as well as the dose of gentamicin they were given. The correct gentamicin dose was calculated for each patient and compared with the dose which had been prescribed and dispensed. The results showed that only $30 \%$ of patients received the correct dose of gentamicin.

\section{Design}

When considering the underlying cause of this problem, it became clear that the calculations involved for calculating gentamicin accurately were too complex in the busy environment that healthcare professionals work in. The reliance of mentally calculating the dose had to be overcome. The most obvious way was to automate the calculations and to generate the correct dose based on the inputting of certain parameters. A gentamicin calculator was developed which allowed users to input a patient's height, weight, age, creatinine and gender, which would then give a result by pressing a 'dose' button.

\section{Strategy}

\section{PDSA Cycle 1}

The calculator was initially shown to the consultant microbiologist in charge of antibiotics within the trust as well as the anti-microbial pharmacist. It was rigorously tested looking at its accuracy of calculation. Whilst being satisfied with the accuracy of the software they 


\section{PDSA Cycle 2}

The calculator was subsequently trialed on the orthopaedic wards with the SHOs and house officers after it had an interface redesign. The feedback from the testing was positive, in that calculation time significantly improved and there was much reliance on the brain for calculation. A useful suggestion, which arose from the testing, was that the calculator should have a height and weight unit converter, which would allow easy conversion of units. The units required for a patient's weight is kilograms and for the height it is feet and inches. Patients often measure their weight in stones and often do not know their height, or know it in metres. The converter would help to overcome this problem.

\section{PDSA Cycle 3}

In the third PDSA cycle the feedback for users was that the height and weight converter improved the user experience and made it very easy for them to convert units. Another issue which arose from this PDSA cycle was that users were not clear about when to take levels from the messages that the calculator was outputting.

\section{PDSA Cycle 4}

The information messages regarding taking levels outputted by the calculator were corrected and it was trialed again with extremely positive and no negative feedback.

\section{PDSA Cycle 5}

The calculator was then trialed for two months on the Surgical Assessment Unit with all gentamicin requests being calculated using the calculator. There was no negative feedback, rather the nursing staff felt empowered to be able to use the calculator to check doctors' prescriptions on the chart.

\section{PDSA Cycle 6}

The calculator was rolled-out within the whole hospital after the two months of testing on the Surgical Assessment Unit. Postmeasurement was carried out six months after rolling the calculator out. See supplementary file: CalcScreenshots.docx

\section{Post-Measurement}

Post-intervention measurement was again for the sake of comparison consistency, carried out with fifty patients on the surgical wards. Again, prophylactic doses of gentamicin were omitted. The findings were that gentamicin had been correctly prescribed in $92 \%$ of cases, demonstrating a $300 \%$ improvement in dosing.

\section{Lessons}

We learnt a number of lessons from carrying out this project:

- Small steps of change are useful when testing an intervention.

- When designing an intervention, consider the wider range of healthcare professionals that the intervention could impact upon.

- Healthcare professionals are not lazy or unintelligent people, the system that they work within limits their time, keeps them busy and makes them tired. One could not expect an individual not to make mistakes in this high-pressured environment, so interventions should seek to eliminate the human factors, which may have a bearing on the process.

\section{Conclusion}

Instituting the intervention was an extremely positive action as it has significantly improved prescribing of gentamicin. Anecdotally, the time taken for prescribing decreased, this would have been a good process measure to measure. The number of incorrect prescriptions decreased, meaning that patients were receiving safer care and the amount of gentamicin being wasted from overdosing was being saved. What is clear is that in a busy environment such as a hospital, its workers need to be aware that they systems that they work within are in need of constant refinement to optimise working conditions and minimise the potential for harm. The introduction of the calculator has clearly had a significantly beneficial effect, but should not make individuals complacent. There is always a need to refine and optimise systems and this project has demonstrated just that. 